\title{
ASTROMETRY WITH THE SPACE TELESCOPE WIDE-FIELD/PLANETARY CAMERA
}

\author{
W. A. Baum
}

Lowell Observatory

Although the Space Telescope Wide-Field/Planetary Camera is not primarily an astrometric instrument, it is expected to have some astrometric capability. Moreover, one of its possible astrometric applications is of unusually high scientific importance, namely, an attempt to detect the presence of planets around nearby stars. Let me therefore adopt that application as an example for discussing the anticipated astrometric performance of the camera system. It will be expeditious to make use of some diagrams that I have presented previously elsewhere, so part of the story may sound familiar (Baum 1979a, 1979b, 1980a, 1980b; Baum, Thomsen, and Kreidl 1981).

Several methods for the detection of extra-solar planets have been discussed in the literature. Direct image detection may ultimately be possible from space. For the Space Telescope, however, image detection puts formidable demands on optical and detector performance, and it requires a fortuitously situated planet. Indirect methods aimed at detecting the reflex motion of a star due to the presence of planets seem to hold more promise if the search is to be systematic. These methods include spectroscopy, interferometry, and astrometry. Of these, astrometry appears the most likely to benefit from the Space Telescope.

Figure 1 shows the optical configuration of the Wide-Field/Planetary Camera. The unique element is the pyramid mirror, which lies at the $f / 24$ focal plane of the Space Telescope. It has four slightly curved faces (only two of which are illustrated in this sketch) splitting the image into four equal quadrants and reflecting them simultaneously into four identical channels with relay optics and CCD image detectors. For wide-field imaging, the relays reduce the beams to $f / 12.9$, resulting in a total field coverage (all four CCDs) of $2.7 \times 2.7$ arcminutes.

Whenever desired, the pyramid can be turned $45^{\circ}$ about the input optic axis so as to throw the four beams into four other channels with relay optics producing $f / 30$, resulting in a total field coverage of $1.1 \times 1.1$ arcminutes. This portion of the system, known as the "Planetary Camera," utilizes the full expected resolution of the Space Telescope. In other words, the complete camera system includes eight relays and eight CCDs, four for f/12.9 wide-field imaging and four for f/30 high-resolution imaging. The so-called Planetary Camera at $f / 30$ is not at all limi ted to bright objects. It can be used for long exposures on faint objects just as the f/12.9 WideField Camera can. Thus, for any astronomical purpose such as the planetary detection problem, one can freely consider the tradeoff between field coverage and resolution when deciding which focal ratio to choose.

Figure 2 illustrates how the total $24 \times 24-\mathrm{mm}$ field is covered by four thinned Texas Instruments CCDs, each having $800 \times 800$ pixels $15 \mu \mathrm{m}$ square. By providing a slight overlap of CCD coverage at the junctions, there will be no gaps, and it will 
be possible to tie the four sub-fields together geometrically by suitable calibration. However, this joining of the four sub-fields cannot be expected to have milliarcsecond stability, so astrometry should be limited to data from a single CCD. The image scales are 102 milliarcsecond per pixel at $\mathrm{f} / 12.9$ and 44 milliarcsecond per pixel at $f / 30$.

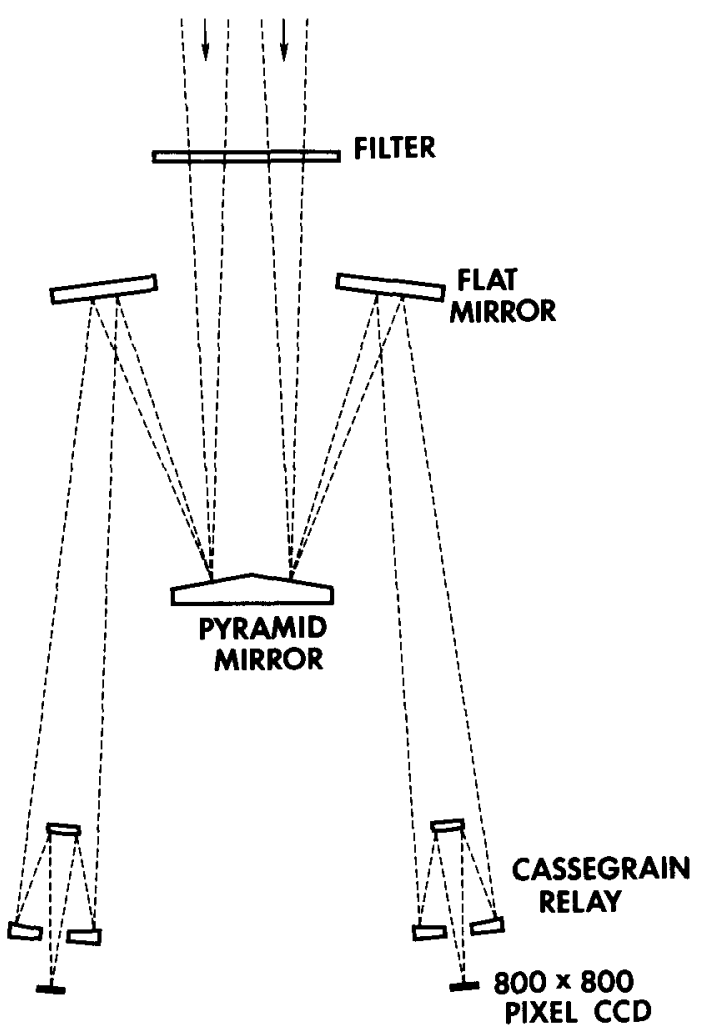

Figure 1. Optical configuration (not to scale) of the Space Telescope Wide-Field/ Planetary Camera. This sketch shows two of the eight CCDs, four of which are for the recording of images relayed at $f / 12.9$ and four for images relayed at $f / 30$.

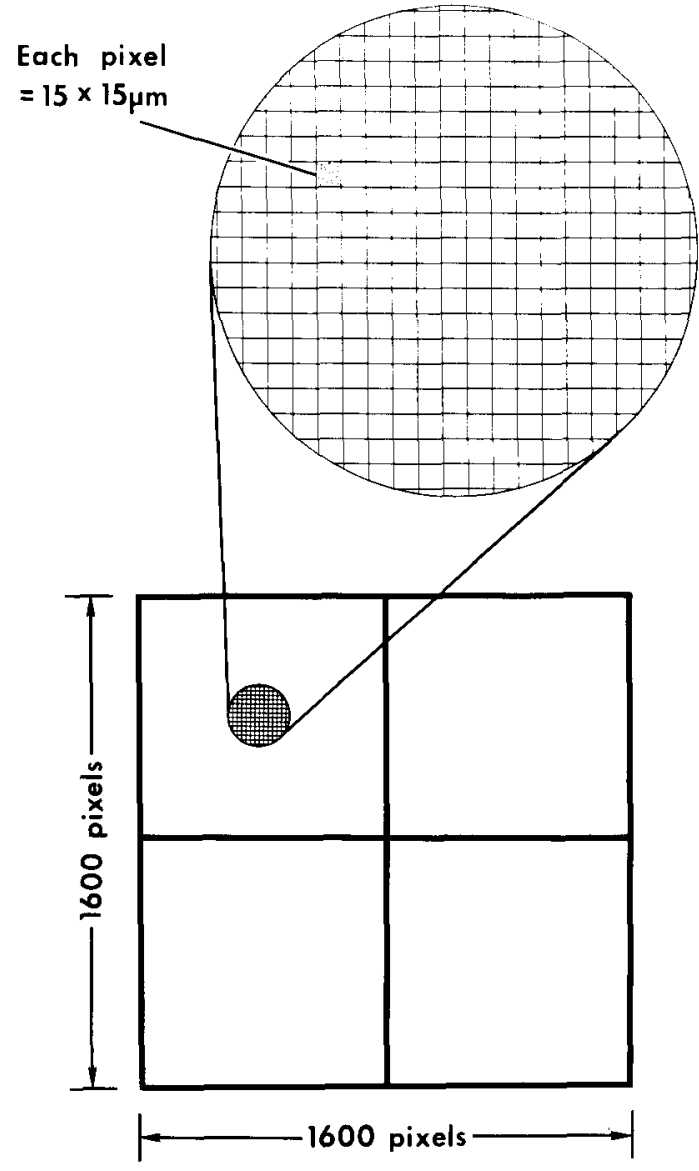

Figure 2. Image field covered by four Texas Instruments CCDs fed by the optical system sketched in Figure 1. Each CCD has $800 \times 800$ pixels $15 \mu \mathrm{m}$ square, so the total field area is $24 \times 24 \mathrm{~mm}$.

CCDs have very high quantum efficiency over a broad spectral range. For the planetary detection problem, the consequence of this high quantum efficiency is that milliarcsecond astrometry is theoretically possible for very faint stars (beyond 20th magnitude), ensuring the existence of at least a few usable reference stars within the field of view in most cases. An important virtue of faint reference stars is that their intrinsic positional variations are typically small so that they provide a reference frame definable at the milliarcsecond level. In this respect, the WideField/Planetary Camera System has an advantage over the Fine-Guidance system; it is less vulnerable to "cosmic errors." 


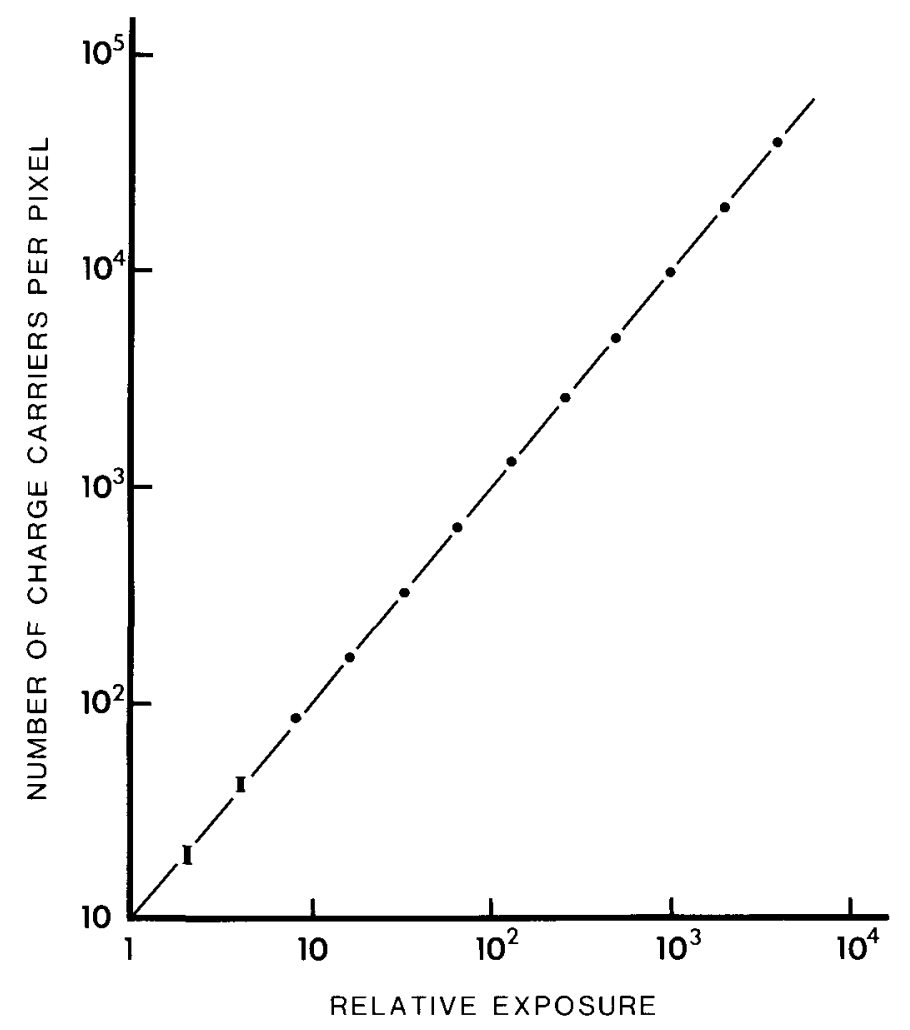

Figure 3. Photometric linearity of a typical 50-pixel sample near the center of a TI $800 \times 800$-pixel CCD. Except for the lowest two points (with indicated error bars), the standard errors are smaller than the radii of the plotted points. No departure from linearity can be seen over three orders of magnitude.

As shown in Figure 3, another important property of CCDs is their photometric linearity. For astrometry this means that the centroid of a star image is not dependent on stellar magnitude, despite optical aberrations and guiding errors; i.e., there is no "magnitude equation." Each star image produces a mound of charge carriers a few pixels wide on the CCD. Therefore, the centroiding of a star image will be precise if there are enough charge carriers in the image for the relative statistical uncertainty $\left(n^{-\frac{1}{2}}\right)$ to be small, if there are enough pixels within the star image to sample its profile adequately, and if we are able to calibrate relative pixel sensitivities accurately.

For astrometry, there is an optimum relationship between the size of a star image and the size of a pixel. If the effective focal length is too short so that the optical image is excessively compressed, the point-spread function (i.e., the star image profile) will occupy too few pixels to determine its centroid precisely. On the other hand, if the effective focal length is too long so that the optical image is excessively magnified, the point-spread function will occupy more pixels than necessary to determine its centroid precisely, while the CCD will cover too small a field in the sky to provide enough reference stars for astrometry. The optimum relationship, of course, lies somewhere between these two situations; and it turns out that the $f / 30$ and $f / 12.9$ systems fall in the desirable range of pointspread functions and field sizes. 


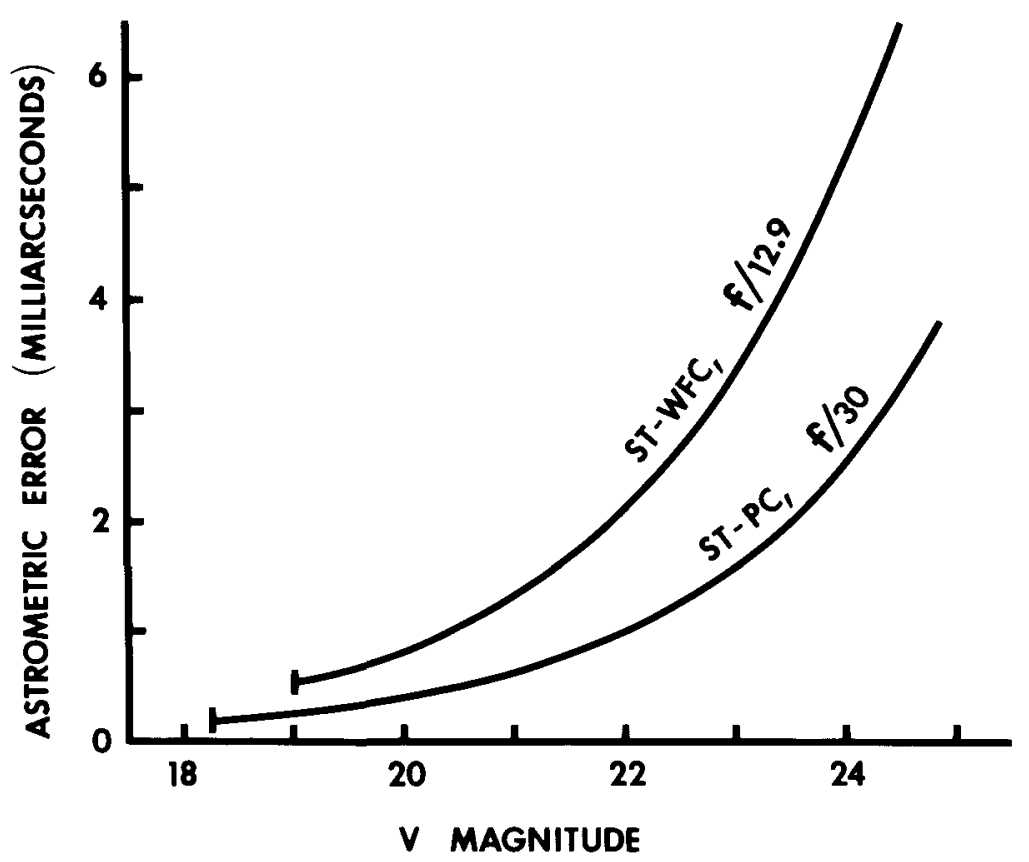

Figure 4. Precision with which the centroids of star images should be determined from 1000-second exposures with the Space Telescope CCD camera system. Bars at the left-hand ends of these curves indicate approximate saturation magnitudes.

Using a very simple "worst-case" model, I have calculated the expected astrometric error shown in Figure 4 as a function of star magnitude for the two CCD cameras and for exposures of 1000 seconds. This plot indicates that an accuracy of about $1 \mathrm{milliarcsecond}$ should in principle be achieved down to 22 nd magnitude with the $\mathrm{f} / 30$ planetary camera, whereas the $f / 12.9$ wide-field camera has a 2-milliarcsecond error at that magnitude. However, the $f / 12.9$ camera covers a field five times larger in sky area, so more reference stars would be available. Bars at the left-hand ends of the curves here indicate approximate saturation magnitudes for 1000-second exposures. For shorter exposures, these curves (and the saturation exposures) shift toward the left.

Many of the nearby stars that are astrometrically desirable to test for the presence of planetary systems are too bright. They would saturate the CCD within any reasonable exposure time that provides enough reference stars. Some of the planetary system candidates therefore have to be separately attenuated without introducing variable astrometric errors. We are currently making an effort to produce a suitable attenuation factor ( $\sim 6$ mags) at the $\mathrm{f} / 24$ focal plane by providing a tiny bare spot (non-aluminized) on one face of the pyramid mirror and putting an antireflection coating on it. If successful, this spot will enable us to observe planetary system candidates as bright as 13th magnitude while being able to make good astrometric use of reference stars down to 22nd magnitude. Candidates as bright as 10th magnitude should be included in our selection exercise, however, because there are fortuitous cases possessing enough reference stars without including those so faint as 22 nd magnitude; i.e., exposures much shorter than 1000 seconds can be used for such cases.

My remarks thus far have assumed that relative pixel sensitivities can be calibrated with as much accuracy as needed to avoid centroiding errors. Probably they 
can, but it is not yet fully certain. Figure 5 illustrates the problem for the particular type of CCD to be installed in the Space Telescope camera. It shows a CRT display (which accounts for the barrel distortion) of a $128 \times 128$-pixel sample of an exposure to faint uniform 117 umination; i.e., it shows about one-sixth of the width of the CCD frame, and it is a low-charge-level "flat field." One sees several columns of pixels that are less sensitive (darker) than the rest. They are known as "deferred charge" columns, and they possess a non-linear toe at very low charge levels. This departure from strict linearity in those particular columns complicates the calibration process, and we are not yet sure of its stability. For astrometry, of course, the problem could largely be circumvented by avoiding the placing of star images on deferred-charge columns.

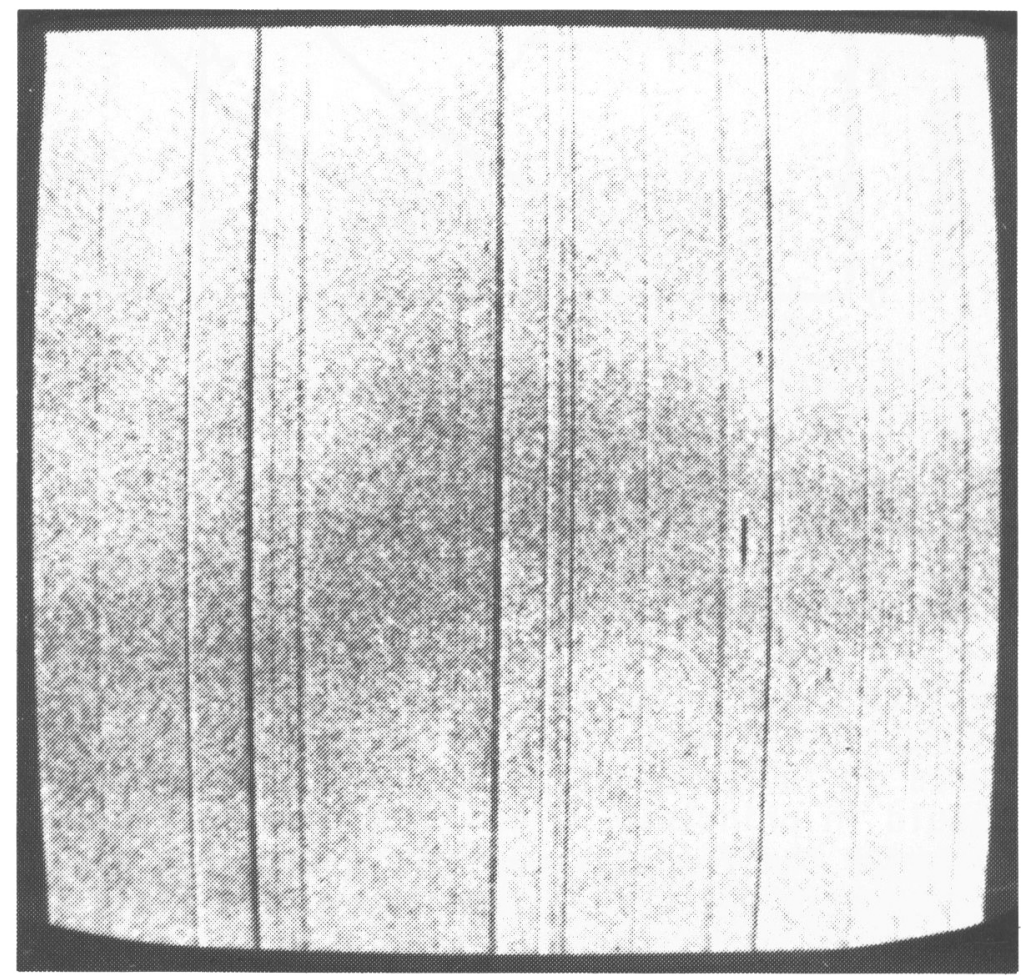

Figure 5. CRT display of a $128 \times 128$-pixel area from the center of a TI $800 \times 800$-pixel CCD exposed to uniform illumination at a low signal level. Some of the columns that appear darker than others suffer from a low-level non-linearity discussed in the text.

Except for those columns, local variations of sensitivity on the CCD are not particularly greater than those on a photographic plate. One should not be misled by blotchy looking reproductions (that CCD critics are fond of showing) in which the contrast has been greatly stretched. There is, however, a large-scale tapering off of sensitivity toward the corners. In any case, all non-uniformities except deferred-charge columns can be calibrated with precision and without special procedures.

Since CCDs are thin membranes, they are not perfectly flat surfaces, and they may flex slightly in the environment to which they are subjected on ground-based telescopes. At fast focal ratios, such behavior could be astrometrically troublesome. 
Aboard Space Telescope, however, the environment is somewhat more benign and the focal ratios are not fast, so we doubt that non-flatness and flexing will be serious problems.

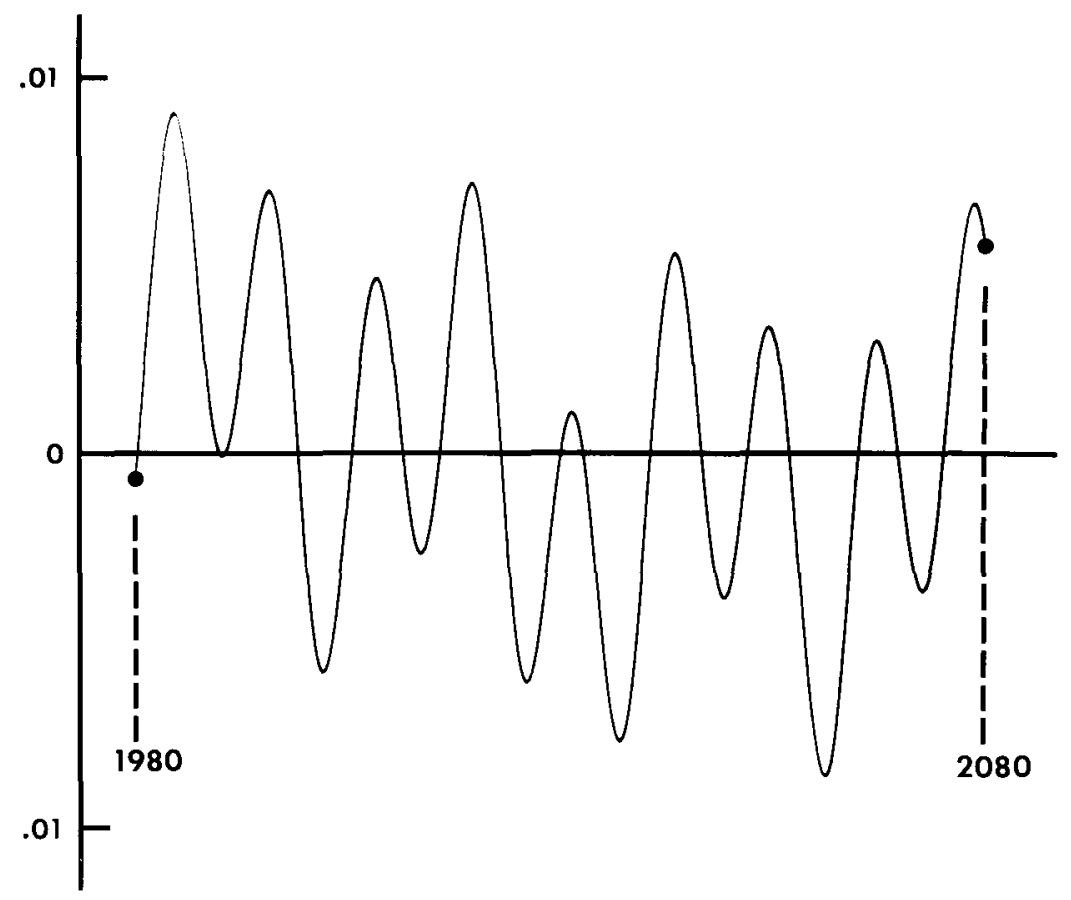

Figure 6. Positional wobble of the Sun due to the major planets of the Solar System. Displacement of the Sun in astronomical units (ordinate) is plotted against time for a 100-year interval starting in 1980.

Let us now consider how much positional wobble of a candidate star we are looking for and therefore how many candidates are in reach of space Telescope instruments. If one plots the actual wobble of the Sun due to the planets of our own Solar System, it is not a simple sine wave with a twelve-year period due to Jupiter. As Figure 6 shows, it is a surprisingly complex curve in which all of the major planets play significant roles. The time interval plotted in Figure 6 is 100 years and the peakto-peak amplitude is about 0.018 a.u. Based on solar wobble, the existence of a planetary system around our sun might be detected by distant alien observers in a few years, but the specific contents of our Solar system would evidently take them many decades to figure out.

Nevertheless, the contribution of Jupiter is a good yardstick for the typical amplitude of variation within any decade-long interval. So as a criterion for the astrometric detectability of other planetary systems, I have imagined each nearby star to possess a hypothetical "Jupiter" (a planet of Jupiter's mass and orbital semi-major axis) and have calculated the resulting amplitude of positional oscillation of the star that would be expected. Since the overwhelming majority of nearby stars are main-sequence dwarfs, I have used a simple linear mass-luminosity relation to translate absolute magnitudes into approximate masses. The inappropriateness of this relation for giants and subgiants (which comprise most of the remaining $6 \%$ of nearby stars catalogued) can be shown to have rather little bearing on the inferred number of good astrometric candidates for planetary system detection. 


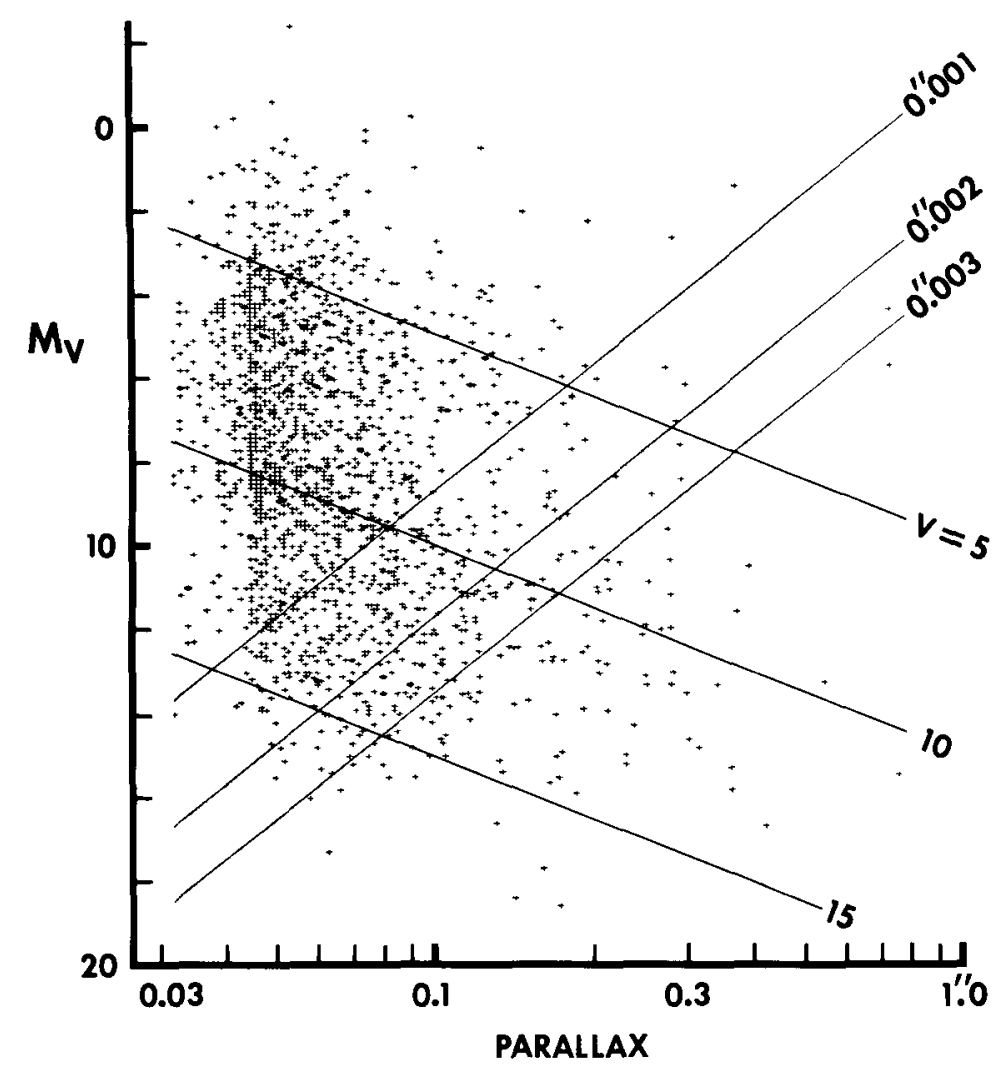

Figure 7. Absolute magnitude distribution of nearby stars in W. Gliese's 1969 catalog as a function of parallax. Sloping lines represent instrument performance parameters defined in the text. Based on those, the best candidates for planetary system detection with the ST camera lie just below the middle of the diagram.

Figure 7 is a plot of absolute visual magnitude $M_{V}$ against parallax for the stars in Gliese's catalog, updated with some Naval Observatory data. My "Jupiter" condition is represented by the family of upward sloping lines. Specifically, they represent half-amplitudes of wobble of 1,2 , and $3 \mathrm{milliarcseconds,} \mathrm{and} \mathrm{one} \mathrm{may}$ think of them as approximate upper limits for stars whose "Jupiters" could realistically be detected by instruments having astrometric thresholds of 1,2 , or 3 milliarcseconds. The downward sloping lines represent the loci of stars having the indicated values of apparent $V$ magnitude.

Planetary system candidates accessible to the Space Telescope Wide-Field/ Planetary Camera should be selected from among the approximately 70 stars to the right of the 0.003 -arcsecond line and to the left of the $V=10$ line. Our best candidates may be among those near that intersection, so that we avoid the stars of lowest mass (those near the bottom of the diagram), whose planets may have inconveniently long orbital periods. Final selection of the prime candidates must, as mentioned earlier, take account of the number and distribution of suitable reference stars. 


\section{$\underline{\text { References }}$}

Baum, W. A. 1979a, in NASA CP-2111, IAU ColZoquium 54 (Eds: M. S. Longair and J. W. Warner), U. S. Government Printing Office, pp. 90-95.

Baum, W. A. 1979b, in European SatelZite Astrometry (Eds: C. Barbieri and P. L. Bernacca), University of Padova, pp. 125-130.

Baum W. A. 1980a, Celestial Mechanies, 22, 183-190.

Baum, W. A. 1980b, in Strategies for the Search for Life in the Universe (Ed: M. D Papagiannis), D. Reidel, Dordrecht, pp. 163-166.

Baum, W. A., Thomsen, B., and Kreidl, T. J. 1981, in Solid-State Images for Astronomy (Eds: J. Geary and D. Latham), S.P.I.E., in press.

\section{DISCUSSION}

HARRINGTON: Are you aware of plans of the WF/PC team to do any thing with respect to binaries?

BAUM: The team has a shopping list, but it is very long, and it includes a11 kinds of science that can be done with the camera. No attempt has been made up to this point to sort out that list and work it down to size. It will have to be cut from its present size by a factor of ten, and whether or not there will be binary stars on it when it comes to that is a decision that has not been made and won't be made for quite some time.

MONET: The vast majority of the astrometric suitability of ST depends on the spot which dims the bright stars. Would you comment?

BAUM: The type of anti-reflection coating which is being used has to be a wide-field coating, because we have to be able to use a reasonable exposure time. The quality of coatings available is good, so we are hopeful, but we are going to have to produce a spot and see how good it is. This problem is not the only reason we want a spot. Every bright galaxy will saturate the nucleus, so we want to be able, for galaxies that are not very far away, to put the nucleus on the spot, so we can detect and see structure in the rest of the body.

CURRIE: A comment on the accuracy requirement. At about one-third of a micron is the point where a number of systematic errors start becoming serious, and that translates into one millisecond.

MCALISTER: What sort of pointing stability will be provided by the fine-guidance system?

FREDRICK: The design was for 0."001, but the budget comes out a little over 0 ".002.

BECKERS: Past space experiments have exceeded their specified angular resolution. Is there a chance that this may happen for ST? If so, is there a danger of undersampling the images, thus reducing your accuracy?

BAUM: The point-spread functions shown in the announcement of opportunity are probably a bit on the optimistic side, because they don't take microstructure of the surface into account. I would be a little surprised if they were exceeded, and if you use these as a base, the $f / 30$ system at least is not undersampling. 